\title{
PAPEL MEDIADOR DO BIG DATA ANALYTICS ENTRE GESTÃO DA CADEIA DE SUPRIMENTOS DE SERVIÇOS E DESEMPENHO OPERACIONAL NA SATISFAÇÃO DO CLIENTE
}

\author{
ROLE OF BIG DATA ANALYTICS FOR CUSTOMER \\ SATISFACTION IN MEDIATING SERVICE SUPPLY CHAIN \\ MANAGEMENT AND OPERATIONAL PERFORMANCE
}

\author{
PAPEL DE MEDIACIÓN DE BIG DATA ANALYTICS ENTRE \\ GESTIÓN DE LA CADENA DE SERVICIOS Y DESEMPEÑO \\ OPERATIVO EN LA SATISFACCIÓN DEL CLIENTE
}

Douglas Vieira Silva

Diretor Financeiro da GDE Bombas e

Equipamanetos Ltda., Brasil

Mestre em Administração de Empresas pela

Universidade Presbiteriana Mackenzie, Brasil

douglas.vieira@gdebombas.com.br

\section{Roberto Giro Moori}

Docente da Universidade Presbiteriana

Mackenzie, Brasil

Doutor em Engenharia da Produçao pela

Universidade de São Paulo, Brasil

roberto.g.moori@gmail.com

\author{
Contextus \\ ISSNe 2178-9258 \\ Organização: Comitê Científico Interinstitucional \\ Editor-Chefe: Diego de Queiroz Machado \\ Editores convidados: Alexandre Graeml; Mônica Cavalcanti \\ Sá de Abreu \\ Avaliação: double blind review pelo SEER/OJS \\ Artigo indicado para fast-track no XLIII Encontro da Anpad \\ (ENANPAD 2019) \\ Recebido em 20/10/2019 \\ Aceito em 04/11/2019 \\ http://dx.doi.org/10.19094/contextus.v17i3.42468
}

\section{RESUMO}

O objetivo do estudo foi examinar o efeito mediador do BDA (big data analytics), como ferramenta, na relação entre gestão da cadeia de suprimentos de serviços e desempenho operacional, na busca pela satisfação do cliente. Para tanto, realizou-se uma pesquisa exploratória do tipo descritiva junto a uma amostra de 125 gestores de empresas de serviços e condomínios residenciais e prediais da região metropolitana de São Paulo. Os dados, tratados pela estatística descritiva e modelagem de equações estruturais, revelaram que o BDA medeia parcialmente a relação entre gestão da cadeia de suprimentos de serviços e o desempenho operacional, que por sua vez, influencia positivamente a satisfação do cliente. A partir desses resultados, pode-se concluir que o BDA é uma importante ferramenta de gestão por facilitar a prestação de serviços em condomínios residenciais ao permitir antever problemas em equipamentos utilizados pelos clientes, como elevadores, bombas hidráulicas, portões automáticos e câmeras de monitoramento de acesso de pessoas nos condomínios, melhorando, assim, o desempenho operacional das empresas prestadoras de serviços condominiais e estendendo-se à satisfação do cliente com a prestação do serviço.

Palavras-chave: Gestão da cadeia de suprimentos de serviços; desempenho operacional; big data analytics; satisfação do cliente.

\footnotetext{
ABSTRACT

The aim of the study was to examine the mediating effect of big data analytics (BDA) as a tool in the relationship between service supply chain management and operational performance in the pursuit of customer satisfaction. To this end, an exploratory descriptive research was conducted with a sample of 125 managers of residential and building service and condominium companies in the metropolitan region of São Paulo. The data, treated by descriptive statistics and structural equation modeling, revealed that BDA partially mediates the relationship between service supply chain management and operational performance, which in turn positively influences customer satisfaction. From these results it can be concluded that BDA is an important management tool for facilitating the execution of service activities in residential condominiums in order to foresee problems in equipment used by customers, such as elevators, hydraulic pumps, automatic gates and access monitoring cameras,
} 
thereby improving the operational performance of condominium service companies and contributing customer satisfaction with service provision.

Keywords: Service supply chain management; operational performance; big data analytics; customer satisfaction.

\section{RESUMEN}

El objetivo del estudio fue examinar el efecto mediador del análisis de big data (BDA) como una herramienta en la relación entre la gestión de la cadena de suministro de servicios y el rendimiento operativo en la búsqueda de la satisfacción del cliente. Con este fin, se realizó una investigación descriptiva exploratoria con una muestra de 125 gerentes de empresas de servicios residenciales y de construcción y condominios en la región metropolitana de São Paulo. Los datos, tratados mediante estadísticas descriptivas y modelos de ecuaciones estructurales, revelaron que BDA media parcialmente la relación entre la gestión de la cadena de suministro de servicios y el rendimiento operativo, que a su vez influye positivamente en la satisfacción del cliente. A partir de estos resultados, se puede concluir que el BDA es una herramienta de gestión importante para facilitar la ejecución de actividades de servicio en condominios residenciales para prever problemas en los equipos utilizados por los clientes, como ascensores, bombas hidráulicas, puertas automáticas y cámaras de monitoreo de acceso a condominios en condominios, mejorando así el desempeño operativo de las empresas de servicios de condominios y la satisfacción del cliente con los servicios.

Palabras clave: gestión de la cadena de suministro del servicio; rendimiento operativo; análisis de big data; satisfacción del cliente.

\section{INTRODUÇÃO}

A Gestão da Cadeia de Suprimentos (GCS) tem sido objeto de estudos cujos esforços são focados em compreender a indústria de transformação, cabendo destaque para o setor automobilístico. A gestão da cadeia de suprimentos em serviços (GCSS) é tida como extensão da GCS, em que os estudos convergem basicamente para dois fluxos: de materiais e das informações (PIRES; CARRETERO-DÍAZ, 2007; BALLOU, 2007; KRITHIKA et al., 2011). Ainda, a GCSS trata de suprimentos de insumos, peças, materiais e pessoal para desenvolver serviços específicos, apropriados e eficientes aos clientes finais (BALTACIOGLU, et al., 2007; WANG et al., 2015).

Neste sentido, tomar decisões assertivas no processo produtivo inserido no contexto da GCSS reflete na estratégia corporativa, principalmente nos processos de desenvolvimento de produtos e serviços, clientes, produção, fornecimento e logística (SUKATI et al., 2012).

Os resultados alcançados pela organização em seus mais diversificados aspectos operacionais são definidos como desempenho operacional (DO) (COMBS et al., 2005). O desempenho na área de produção e operações pode ser sintetizado em aspectos como qualidade, custos, flexibilidades, dentre outros (FLYNN et al., 2010; MILLER; ROTH, 1994; ROSENZWEIG; EASTON, 2010).

Não obstante o uso de tecnologias da informação como uma ferramenta essencial para gerenciar dados relacionados à agilidade e flexibilidade (PAULRAJ; CHEN, 2007), o BDA (big data analytics) tem emergido como a nova fronteira da tecnologia digital (SANDERS, 
2016), que, além de gerenciar dados, processa e analisa dimensões como volume, variedade, velocidade, veracidade e valor (WAMBA et al., 2017). A utilização do BDA nas organizações tem contribuído para o desenvolvimento de novas estratégias, bem como novas formas administrativas, de inovação de produtos e novas maneiras de criar valor tanto para produtos como em serviços (PORTER; HEPPELMANN, 2015). Entretanto, se faz necessário avaliar em que grau a utilização desta ferramenta tecnológica é capaz de contribuir para que as organizações, por meio das análises dos dados, transformarem as suas operações na GCSS (SANDERS, 2016).

Trilhões de bytes de informação são capturados diariamente por organizações das mais diversas atuações ao redor do mundo. Em geral esses dados são gerados pelos clientes, empresas e fornecedores, em operações logísticas e financeiras, dentre outras fontes das mais diversificadas, como equipamentos e aplicativos em smartphones e notebooks (MANYIKA et al., 2011). Com a disponibilidade de grandes bancos de dados e a evolução tecnológica, permitindo maior funcionalidade de buscas de soluções de compras pelos clientes, as operações das empresas passaram a receber maior atenção da GCSS e, por conseguinte, obter melhores desempenhos operacionais (DO) e maior satisfação do cliente (SC) (PORTER; HEPPELMANN, 2015). Embora SC seja um conceito fundamental da estratégia de marketing e negócios (BOWERSOX et al., 2014), a GCSS é um processo de integração para gerar valor que atravessa as áreas funcionais dentro de empresas individuais e conecta a parceiros além das fronteiras organizacionais, proporcionando a satisfação do cliente (BOWERSOX et al., 2007; BALLOU, 2007).

Baseado nessas premissas (BDA, GCSS, DO e SC), o presente estudo teve como objetivo responder à seguinte questão: o BDA tem efeito mediador na relação entre a GCSS e o DO, subsequentemente, impactando positivamente na SC? Assim examinou-se o efeito mediador do BDA no DO e, consequentemente, na SC, em empresas de prestação de serviços e condomínios residenciais e comerciais.

O artigo está estruturado da seguinte maneira. Após a introdução, é apresentado o referencial teórico. Na sequência, são discutidos os procedimentos metodológicos, seguidos da análise dos dados e resultados. Por fim, são apresentadas as conclusões e sugestões para prosseguimento. 


\section{REFERENCIAL TEÓRICO E HIPÓTESES}

A gestão da cadeia de suprimentos de serviços (GCSS) repousa no paradigma da vantagem competitiva, agregação de valor (PORTER, 1985), e na visão relacional para influenciar a competitividade entre os parceiros da cadeia de suprimentos (DYER; SINGH, 1998). Conforme a visão relacional, uma cadeia de suprimentos de serviços é composta de uma sequência de atividades interdependentes promovidas pela colaboração, que incentiva comportamentos bilaterais e produz benefícios comuns, ao invés de comportamentos individuais de busca de renda que maximiza benefícios próprios da empresa de serviço (CAO; ZHANG, 2011).

A literatura sobre gestão da cadeia de suprimentos é representada por múltiplas perspectivas. Este estudo examina a gestão da cadeia de suprimentos a partir de três perspectivas: da vantagem competitiva conjunta (PORTER, 1985: CAO; ZHANG, 2011); da teoria de custos de transação (WILLIAMSON, 1999) e da visão relacional (DYER; SINGH, 1998). Assim, de um lado; a visão relacional da gestão da cadeia de suprimentos em serviços ajuda a reduzir os custos do oportunismo e monitoramento, que são inerentes às transações de mercado, aumentando a probabilidade de que os parceiros se comportem no melhor interesse da parceria. De outro, a vantagem competitiva conjunta é proveniente de um benefício comum entre parceiros colaborativos, que possibilita agregar valor e melhor atendimento ao cliente.

\subsection{Efeito direto: a influência da gestão da cadeia de suprimentos de serviços no desempenho operacional}

Existe consenso de que os serviços são responsáveis por uma expressiva representatividade no PIB mundial. Todavia, estudos realizados evidenciam a existência de grandes oportunidades de aprimoramento neste meio, que podem ser exploradas pelas organizações em um amplo contexto dos negócios empresariais (ZEITHAML; BITNER, 2003).

Tanto no campo das pesquisas quanto das práticas gerenciais, a GCSS não possui modelos gerais largamente aceitos, existindo uma representatividade maior da utilização das cadeias de suprimentos orientadas aos produtos, embora recentemente haja tentativas da formalização de cadeias de suprimentos de serviços (CHOU et al., 2006; BALTACIOGLU et al., 2007; FAVARETTO, 2012). Neste caminho, um modelo para as cadeias de suprimentos de serviços 
foi desenvolvido com base nas melhores práticas de modelos tradicionais para as cadeias de manufatura, mas voltado especificamente para os serviços (ELLRAM et al., 2004).

Assim, a GCSS tem sido apresentada como contraponto às tradicionais cadeias de suprimentos industriais convencionais existentes (STARR, 2005). As cadeias de suprimento convencionais são responsáveis por gerar um conjunto de atividades e processos com o objetivo de fazer com que insumos sejam adquiridos, transformados em bens e serviços e entregues aos seus consumidores finais (COSTA; TORRES, 2014). Todavia, nos últimos anos, um meio que vem sendo utilizado com o objetivo de buscar a redução de custos e desenvolver melhoria do nível de serviços é a GCSS. É notório que uma gestão eficaz e eficiente da cadeia de suprimentos de serviços traz diversos benefícios para as empresas parceiras e influência no desempenho operacional das organizações (BALLOU, 2007; BOWERSOX et al., 2007).

Os estudos apresentados até o presente momento já destacam o relacionamento entre esses construtos, contudo, buscou-se examinar com mais sensibilidade o papel do BDA como ferramenta, bem como o seu impacto na GCSS, no DO e na SC.

Assim, é de se esperar que:

$\mathrm{H}_{1}$ : A Gestão da Cadeia de Suprimentos de Serviços impacta positivamente no Desempenho Operacional.

\subsection{Efeitos indiretos}

\subsubsection{A influência do big data analytics na gestão da cadeia de suprimentos de serviços}

Nos últimos anos o tema BDA tem chamado a atenção não somente dos profissionais do mercado, cada vez mais focados em inovação, mas também dos acadêmicos, como se pode notar pelo volume crescente das publicações. Ao vivenciar a explosão de dados (CHOI; LAMBERT, 2017), um número significativo de empresas tem desenvolvido projetos relacionados ao BDA (big data analytics) (KIRON et al., 2014), que considera-se ter grande potencial para revolucionar os mais diversos campos do conhecimento e em diferentes ambientes de negócios (CHEN; ZHANG, 2014). 
A definição do termo BDA (big data analytics) apresenta diversos aspectos que muitas vezes não convergem, uma vez que cada organização leva em conta a sua perspectiva para definir este construto. É importante que os conceitos de BDA sejam claros e representativos para que o trabalho alcance a sua finalidade. Nesse contexto, a GCSS ao gerar milhões de informações estimula as organizações a disporem de estruturas capazes de armazenar esses dados, de tal modo a extrair valor (ITTMANN, 2015). Assim, é de se esperar que:

$\mathrm{H}_{2}$ : O Big Data Analytics impacta positivamente na Gestão da Cadeia de Suprimentos de Serviços.

\subsubsection{A influência do big data analytics no desempenho operacional}

A tecnologia digital tem contribuído para que significativas mudanças ocorram impactando diretamente a gestão da cadeia de suprimentos (SANDERS, 2016). Inovações aplicadas na gestão da cadeia de suprimentos de serviços permitem que as organizações se mantenham atualizadas e competitivas em relação ao mercado em que atuam (RODRIGUEZ; CUNHA, 2018). Para que isso ocorra, se faz necessário que as empresas incorporem tecnologias existentes nos mais diversos processos organizacionais, de modo que as permitam alcançar a competitividade e a sustentabilidade ao longo da cadeia de suprimentos (MAJEED; RUPASINGHE, 2017).

A inovação na GCS é um aspecto indispensável para que as empresas de transformação e de serviços possam acessar novas oportunidades e manter sua vantagem competitiva em um mercado cada vez mais dinâmico (LI; LI, 2017). Um estudo de Rossmann et al. (2018) sugere que o BDA melhora as previsões de demanda, reduz estoques de segurança e melhora a administração e o desempenho operacional dos fornecedores. Assim, é de se esperar que: $\mathrm{H}_{3}$ : O Big Data Analytics impacta positivamente no Desempenho Operacional. 


\subsubsection{A mediação do big data analytics na relação entre gestão da cadeia de suprimentos de serviços e desempenho operacional}

O desempenho organizacional é resultado da cooperação entre as diversas áreas da organização e de seus pares (CARVALHO, 2005). A troca de informação e a gestão compartilhada permitem que os fatores de desempenho operacional da cadeia possam ser continuamente avaliados, contribuindo para maior assertividade das decisões estratégicas em relação aos produtos e serviços comercializados pela organização. A gestão da cadeia de suprimentos vem sendo muito utilizada pelas empresas com o objetivo de desenvolver melhores indicadores de competitividade (PIRES, 2013).

Os gestores têm vivido momentos de tensão e conflitos, ao enfrentarem o processo de transformação na cadeia de suprimentos, decorrente de inovações em seus sistemas administrativos e produtivos (DONALD et al., 2014). Tais mudanças estão grandemente relacionadas à necessidade de realizar as atividades inerentes à operação de modo customizado e integrado, desde a aquisição de matérias-primas até o atendimento do cliente final. Áreas como marketing, compras, produção, logística e tecnologia da informação estão envolvidas no processo, de forma completamente integrada, em toda a cadeia de suprimentos (DONALD et al., 2014). Por conta disso, as empresas recorrem aos seus bancos de dados para assegurar que seus esforços e empenho terão sucesso para atender as necessidades do mercado. Assim, é de se esperar que:

$\mathrm{H}_{1 \mathrm{a}}$ : Big Data Analytics medeia a relação entre Gestão da Cadeia de Suprimentos de Serviços e Desempenho Operacional.

\subsubsection{A influência do desempenho operacional na satisfação do cliente}

A satisfação do cliente (SC) está relacionada ao desempenho diante das expectativas dos seus clientes (KOTLER, 2000), segundo a qual, “a satisfação consiste na sensação de prazer ou desapontamento resultante da comparação do desempenho (ou resultado) percebido de um produto em relação às expectativas do comprador”.

Existe uma quantidade significativa de estudos que aborda o tema satisfação do cliente aliado à qualidade do serviço, apresentando um alinhamento entre a percepção do cliente em 
relação aos serviços prestados e as suas expectativas. É notória a existência de certa disparidade entre os conceitos de qualidade percebida e satisfação dos clientes, não sendo possível encontrar unanimidade na literatura (MILAN et al., 2015). Diante disso, a percepção do cliente quanto aos serviços prestados é resultado na satisfação (BLOEMER; KASPER, 1995).

Zeithaml e Bitner (2003) argumentam que a satisfação é uma avaliação feita pelo cliente no que se refere a um produto ou serviço, levando em conta as necessidades e expectativas do próprio cliente. A falha em ir ao encontro das necessidades e expectativas dos clientes é pressuposta como causadora da insatisfação com o produto ou serviço.

Diante desse contexto, se faz necessário que as empresas prestadoras de serviços sejam capazes de monitorar os seus indicadores, constantemente, buscando aprimorar os seus recursos e serviços, contribuindo para melhorar o desempenho na prestação de serviços e atividades correlatas para, consequentemente, obterem resultado positivo quanto à satisfação de seus clientes (ANDERSON, 1998; MILAN; DE TONI, 2012). Acompanhar os níveis de satisfação do cliente em relação as suas expectativas e percepções, levando em conta as suas necessidades e desejos faz a diferença na busca da excelência em serviços.

Segundo Kotler (2000), a SC está diretamente relacionada ao desempenho das organizações quanto aos produtos e ou serviços por elas oferecidos. Esta satisfação está relacionada à sensação de prazer ou desapontamento que determinado cliente tem em relação à prestação de serviços quando o desempenho (ou resultado) percebido é comparado com a expectativa do comprador (KOTLER, 2000). Assim, é de se esperar que:

$\mathrm{H}_{4}$ : O Desempenho Operacional impacta positivamente na Satisfação do Cliente

\section{PROCEDIMENTOS METODOLÓGICOS}

\subsection{Natureza e tipo de pesquisa, instrumento de coleta de dados e sujeito da pesquisa}

A pesquisa foi de natureza exploratória do tipo descritiva, precedida de um estudo exploratório junto a seis membros da cadeia de suprimento de serviços, sendo três clientes e três gestores, todos eles relacionados ao uso de BDA (big data analytics) para conhecer com mais profundidade o objeto do estudo e levantar, preliminarmente, medidas para desenvolver o instrumento de coleta de dados (MALHOTRA, 2019). O estudo resultou em um conjunto de 
assertivas que ajudou na construção da primeira versão do questionário. Após, seguindo as recomendações de Dillman (2000), o questionário foi submetido a pré-testes junto a dez gestores usuários de big data analytics na cadeia de suprimentos de serviços para testar o enunciado, a sequência, o formato, leiaute e instruções. Ao final, o estudo exploratório possibilitou avaliar, segundo a abordagem de Hair et al. (2014), a correspondência das medidas a serem incluídas na escala múltipla com sua definição conceitual, garantindo, assim, que a seleção das medidas e escalas abordasse não apenas questões empíricas, mas também incluísse questões práticas e teóricas discutidas no referencial. Ainda, para o instrumento de coleta, escolheu-se a escala par, do tipo Likert, composta de 6 pontos, de 1 a $6(1=$ Discordo Totalmente e $6=$ Concordo Totalmente) por desejar uma resposta que impossibilitasse que o respondente se posicionasse de forma neutra. Essa escala, segundo Toni e Mazzon (2014), permite avaliar o sentido (discordância ou concordância) com o item e a intensidade ou a dosimetria (pouco, muito ou total) da escala de discordância ou concordância.

Assim, construiu-se o questionário final, único, composto de três blocos. O primeiro e o segundo blocos referiram-se a dados dos respondentes e das empresas. No terceiro bloco foi solicitado aos respondentes que assinalassem o seu grau de discordância/concordância em relação às oito medidas de cada constructo: GCSS, BDA, DO e SC. A razão de se adotar um questionário único a ser respondido pelos membros da cadeia de suprimentos de serviços se deveu à complexidade envolvida no entendimento da cadeia de suprimentos, em que uma empresa pode ser, simultaneamente, tanto cliente como fornecedora. Por exemplo, um cliente pode adquirir um equipamento de um fornecedor e, ao mesmo tempo, vender componentes para esse fornecedor (BROWN et al., 2006).

Em seguida foi selecionada, por acessibilidade, uma amostra composta de 125 respondentes entre empresas de prestação de serviços condominiais e condomínios residenciais e comerciais da região metropolitana de São Paulo para envio dos questionários. Um dos critérios de seleção dessas empresas foi a obrigatoriedade da utilização do BDA em pelo menos um dos seus processos: de processamento do pedido de serviços, monitoramento, acesso de pessoas, armazenagem, estocagem ou transporte. Ainda, foi solicitado aos respondentes informar a plataforma de BDA utilizada: privada, pública ou própria.

Definida a amostra, foram considerados como sujeitos da pesquisa, os gestores das empresas relacionadas à utilização do BDA e que compõem a GCSS. Os questionários foram enviados por correio eletrônico, utilizando-se a ferramenta Google Forms para a coleta de dados. 


\subsection{Tratamentos dos dados, delimitação do estudo e limitação do método}

Para o tratamento dos dados coletados, incialmente, utilizou-se da estatística descritiva para examinar os dados em relação à discrepância, valores atípicos e dispersão da distribuição dos dados coletados. Nesse último caso, foram utilizados critérios para classificação do coeficiente de variação $(\mathrm{CV})$, que seguiram parâmetros usuais em ciências sociais e aplicadas, considerando-se que CV < 15\% representa baixa dispersão, 15\% < CV < 30\%, média dispersão e CV > 30\%, elevada dispersão da distribuição dos dados coletados (PRATES, 2017). Encerrada a análise da estatística descritiva, prosseguiu-se em validar as medidas e escalas; analisar a significância estatística e avaliar os ajustes das relações estruturais do modelo de mensuração.

Para validar as medidas e escalas das estruturas dos constructos do modelo de mensuração, os dados foram submetidos à análise fatorial exploratória e confirmatória para depuração das medidas e de suas respectivas escalas. Foram consideradas como válidas as medidas com cargas fatoriais iguais ou superiores a 0,7 . Foi aplicado o teste de unidimensionalidade, por meio do coeficiente Alfa de Cronbach, cujos valores de referência devem estar acima de 0,70 (HAIR et al., 2014). Para a avaliação da confiabilidade composta, foi considerado como mínimo aceitável o valor de 0,7 (CHIN, 1998). A validade de conteúdo foi estabelecida na pesquisa exploratória em que foi avaliada subjetivamente a correspondência entre as medidas individuais e o conceito por meio de julgamentos de especialistas e pré-teste. A validade convergente foi avaliada pelas cargas fatoriais superiores a 0,7 e pela variância média extraída (VME) com valores superiores a 0,5 . Por fim, a validade discriminante se deu por meio das cargas cruzadas, sendo que os indicadores deveriam ser mais altos nas suas respectivas variáveis latentes (CHIN, 1998). Para analisar a significância estatística das relações estruturais do modelo de mensuração $(\alpha \leq 0,05)$, utilizou-se a técnica da modelagem em equações estruturais, o Partial Least Squares-Path Modeling (PLS-PM).

Para avaliar os ajustes das relações estruturais do modelo de mensuração foram utilizados: a) o coeficiente de determinação $R^{2}$, para o qual Cohen (1988) considera um valor de 0,26 como de grande efeito para a área de ciências do comportamento humano e social e b) o índice de ajuste global, o índice GoF (Goodness of Fit), dado por:

$$
\left(\mathrm{GoF}=\sqrt{\overline{\overline{A V E} * \overline{R^{2}}}}\right) \rightarrow[\text { Equação } 1],
$$

para o qual Wetzels et al. (2009) sugerem o valor de 0,36 como o valor mínimo aceitável. 
Complementarmente, foram utilizados dois outros indicadores de qualidade de ajuste dos modelos: o indicador de relevância ou validade preditiva $\left(\mathrm{Q}^{2}\right)$, também denominado de indicador de Stone-Geisser, para o qual deve ser obtido valor maior que zero para cada constructo; e o indicador de tamanho do efeito $\left(\mathrm{f}^{2}\right)$, ou indicador de Cohen, em que se avalia quanto cada constructo é útil para o ajuste do modelo. Valores $\mathrm{f}^{2}$ de $0,02,0,15$ e 0,35 são considerados pequeno, médio e grande, respectivamente (Hair et al., 2014).

Para testar e tipificar o efeito mediador do fator BDA (big data analytics) na relação entre gestão da cadeia de suprimentos de serviços e desempenho operacional foi utilizado o teste da variância contabilizada (VAF - Variance Accounted For), dada por:

$$
\mathrm{VAF}=\left[\frac{\beta 12 \times \beta 23}{(\beta 12 \times \beta 23)+\beta 13}\right] \rightarrow[\text { Equação } 2],
$$

onde $\beta_{12}, \beta_{23}$ e $\beta_{13}$ são os coeficientes estruturais correspondentes aos relacionamentos entre os constructos [GCSS $\rightarrow$ BDA], [BDA $\rightarrow$ DO] e [GCSS $\rightarrow$ DO], respectivamente. Valores de VAF $>80 \%$, significam mediação total, $20 \% \leq \mathrm{VAF} \leq 80 \%$ significa que a mediação é parcial e VAF < 20\% significa que não existe mediação. Utilizou-se o software SMARTPLS versão 3.0 para tratar os dados coletados.

A principal limitação do método da pesquisa se referiu ao tamanho da amostra. Segundo Hair et al. (2014), para análise de dados multivariados, um dos critérios para definir o tamanho da amostra é que ela seja pelo menos maior do que o número de correlações. Para o estudo com 18 variáveis obtém-se 153 correlações. Como foram obtidas 125 medidas, o tamanho da amostra ficou aquém do mínimo necessário. No entanto, julgou-se satisfatória em decorrência do perfil do sujeito da pesquisa e das suas respectivas empresas, tendo em vista que quantidade significativa dos participantes ocupava cargos gerenciais e que tinha profundo conhecimento das características do mercado-alvo do estudo. No entanto, as inferências sobre o resultado da pesquisa devem ser realizadas com ressalvas.

O escopo do estudo foi transversal e delimitado à cadeia de suprimentos de serviços em empresas de prestação de serviços e condomínios residenciais e comerciais da região metropolitana de São Paulo. Nesse sentido, fizeram parte do estudo prestadores dos mais diversos serviços em: elevadores, auto de vistoria do corpo de bombeiros e combate a incêndio, gás, seguros, fachada, bombas hidráulicas, caixas de gordura e passagem, jardinagem, dedetização e controle de pragas, limpeza de caixas d'água, instalações elétricas e hidráulicas, piscinas, controle de acesso e segurança. 


\section{ANÁLISES DOS DADOS E RESULTADOS}

Foram enviados aproximadamente 1.000 questionários, no segundo semestre de 2018, cujos respondentes foram escolhidos por acessibilidade, no segmento condominial. Retornaram 125 questionários (12,5\% do total enviado). Desse total, 88 questionários (70,4\%) referiram-se a prédios condominiais e $37(29,6 \%)$ a empresas de serviços.

O perfil demográfico dos respondentes e das empresas da amostra de 125 respondentes é descrito a seguir.

1) em relação aos respondentes: a) 9,6\% exerciam a função de diretor, 29,6\% de gerentes, 19,2\% de síndicos, 8,8\% de supervisão e 32,8\% eram zeladores; b) 40,8\% possuíam formação superior, 59,2\% ensino fundamental e médio; c) dentre aqueles com formação superior, 58,8\% eram formados em administração, 11,8\% em engenharia e 29,4\% tinham formação em direito, ciências contábeis e outras; d) 56,8\% tinham experiência na função havia mais de 5 anos, 28,0\% entre 2 e 5 anos e 15,2\% menos de 2 anos, e) 42,4\% atuavam em empresas de prestação de serviços a condomínios ou em prédios condominiais havia mais de 5 anos, $28,8 \%$ entre 2 e 5 anos e $28,8 \%$ havia menos de 2 anos.

2) Em relação às empresas de prestação de serviços e prédios condominiais: a) 68,0\% das empresas de prestação de serviços e prédios condominiais tinham mais de 5 anos de existência, $23,0 \%$ entre 2 e 5 anos e 11,0\% até 2 anos; b) 40,5\% exerciam o ramo de administração condominial, 24,3\% de sindicância profissional, 13,5\% de manutenção de bombas hidráulicas, $10,8 \%$ de portaria e limpeza e 10,9\% outros ramos de atividades como manutenção de elevadores e segurança; c) 7,2\% tinham entre 100 e 499 funcionários, 15,2\% entre 20 e 99 funcionários e 77,6\% com até 19 funcionários; d) quanto ao uso de BDA (big data analytics), dos total de 125 respondentes, 30\% (equivalente a 37 respondentes) utilizavam para processamento de pedidos, $20 \%$ (equivalente a 25 respondentes) para armazenagem, $24 \%$ (ou 30 respondentes) para transporte, e $26 \%$ (restante, ou 32 respondentes) não souberam responder; e) quanto ao tempo de utilização do BDA, do total de 37 entrevistados que responderam, 18,9\% o utilizavam havia mais de 3 anos, 48,7\% entre 1 e 3 anos e, 32,4\% até 1 ano; f) quanto ao fornecedor de BDA, do total de 37 entrevistados que responderam, 24,3\% utilizavam a plataforma da Amazon, 10,8\% da Microsoft, 13,5\% da IBM e 51,4\% não souberam responder. $\mathrm{O}$ fato de $51,4 \%$ dos respondentes não souber quem era o fornecedor da plataforma de BDA, não significa que o uso do BDA era precário, dado que, muitos se recusam a responder 
pesquisas sobre BDA devido a compreensão e experiência limitada com BDA (MÜLLER; JENSEN, 2017).

Diante do exposto, acreditou-se que a amostra foi representativa e atendeu ao propósito do estudo tendo em vista que quantidade significativa dos participantes ocupava cargo gerencial ou que permitisse compreender características do ambiente do negócio do estudo, quais sejam, a influência do BDA (big data analytics) em empresas de prestação de serviços a prédios condominiais.

\subsection{Validação das medidas e escalas do modelo de mensuração}

Os resultados da validação das medidas e escalas são mostrados naTabela 1.

Tabela 1 - Validação e Correlação Bivariada entre os Constructos

\begin{tabular}{|c|c|c|c|c|c|c|c|}
\hline \multirow[b]{2}{*}{ VARIÁVEIS } & \multicolumn{3}{|c|}{ Estatística Descritiva } & \multicolumn{4}{|c|}{ Correlação Bivariada } \\
\hline & Média & $\begin{array}{l}\text { Desvio } \\
\text { Padrão }\end{array}$ & $\begin{array}{c}\text { Coef. } \\
\text { Variação }\end{array}$ & BDA & DO & GCSS & $\mathrm{SC}$ \\
\hline BDA - Big Data Analytics & 5,15 & 0,59 & 0,11 & 0,77 & & & \\
\hline DO - Desempenho Operacional & 5,36 & 0,43 & 0,08 & 0,60 & 0,77 & & \\
\hline GCSS - Gestão da Cadeia de Suprimentos de Serviços & 5,32 & 0,46 & 0,09 & 0,53 & 0,55 & 0,79 & \\
\hline SC - Satisfação do Cliente & 5,40 & 0,43 & 0,08 & 0,49 & 0,63 & 0,41 & 0,76 \\
\hline \multicolumn{4}{|c|}{ Número de variáveis originais $\rightarrow$} & 8 & 8 & 6 & 8 \\
\hline \multicolumn{4}{|c|}{ Número de variáveis finais $\rightarrow$} & 6 & 4 & 4 & 4 \\
\hline \multicolumn{4}{|c|}{ Unidimensionalidade (Alfa de Cronbach) $\rightarrow$} & 0,87 & 0,77 & 0,79 & 0,77 \\
\hline \multicolumn{4}{|c|}{ Variância Média Extraída (VME) $\rightarrow$} & 0,60 & 0,59 & 0,62 & 0,58 \\
\hline \multicolumn{4}{|c|}{ Confiabilidade Composta (CC) $\rightarrow$} & 0,90 & 0,85 & 0,87 & 0,85 \\
\hline
\end{tabular}

Nota: Os valores na diagonal da matriz correspondem à raiz quadrada da Variância Média Extraída (VME).

Fonte: dados da pesquisa

Da Tabela 1 percebeu-se que os valores de variância média extraída (VME) foram sempre valores superiores ao mínimo recomendado de 0,5 , denotando a qualidade dos dados e validade convergente. Por sua vez, o valor de alfa de Cronbach apresentou valores superiores a 0,77 
denotando a consistência interna das medidas. A Confiabilidade Composta (CC) apresentou valores superiores ao mínimo aceitável, que é de 0,7 (HAIR, et al., 2014).

Após várias rodadas, de um total de 30 medidas originais, foram validadas 18 , isto é, $60 \%$ das medidas utilizadas apresentaram-se válidas para o desenvolvimento do estudo. Justificativas para a não validação de $40 \%$ das medidas poderiam estar relacionadas à semelhança entre elas, ou ao fato de a medida não ter sido importante na visão dos respondentes. Outro ponto pode ser o fato de a medida ter sido apresentada de maneira genérica, ou seja, envolvendo diversas facetas que não ficaram muito claras para os respondentes.

Prosseguindo na análise, foi verificado que o coeficiente de variação (também conhecidos como desvio padrão relativo) variou entre 0,08 a 0,11 , denotando a concentração dos valores em torno da média e dispondo de baixa dispersão (PRATES, 2017).

Numa análise mais detalhada, na Tabela 2 apresenta-se a estatística descritiva e as cargas fatoriais de cada medida em seus respectivos constructos.

Tabela 2 - Estatística descritiva e cargas fatoriais para cada medida do modelo de mensuração

\begin{tabular}{|c|c|c|c|c|c|c|c|c|c|}
\hline \multirow{2}{*}{\multicolumn{3}{|c|}{ CONSTRUCTO / ASSERTIVAS }} & \multicolumn{3}{|c|}{ Descritiva } & \multicolumn{4}{|c|}{ Cargas Fatoriais } \\
\hline & & & Média & D. $\mathbf{P}$ & C. V & BDA & DO & GCSS & SC \\
\hline \multirow{6}{*}{ 苚 } & BD1 & Permitir analisar gastos de compra & 5,14 & 0,73 & 0,14 & $\mathbf{0 , 7 3}$ & 0,42 & 0,47 & 0,28 \\
\hline & $\mathrm{BD} 2$ & Permitir monitorar processos & 5,18 & 0,73 & 0,14 & $\mathbf{0 , 8 3}$ & 0,47 & 0,45 & 0,41 \\
\hline & BD3 & Permitir monitorar equipamentos & 5,12 & 0,80 & 0,16 & $\mathbf{0 , 8 1}$ & 0,45 & 0,39 & 0,43 \\
\hline & BD4 & $\begin{array}{l}\text { Ter volume de dados para melhorar o } \\
\text { comportamento dos tomadores no } \\
\text { processo de decisão }\end{array}$ & 5,06 & 0,85 & 0,17 & $\mathbf{0 , 7 6}$ & 0,48 & 0,42 & 0,34 \\
\hline & BD5 & $\begin{array}{l}\text { Ter variedade de fontes de informação } \\
\text { para o processo de decisão }\end{array}$ & 5,14 & 0,80 & 0,16 & $\mathbf{0 , 7 9}$ & 0,53 & 0,36 & 0,41 \\
\hline & BD6 & $\begin{array}{l}\text { Ter velocidade de chegada da } \\
\text { informação para a tomada de decisão }\end{array}$ & 5,24 & 0,68 & 0,13 & $\mathbf{0 , 7 2}$ & 0,44 & 0,38 & 0,40 \\
\hline \multirow{4}{*}{ ஜ } & DO1 & Aumentar a eficiência da mão de obra & 5,42 & 0,53 & 0,10 & 0,42 & $\mathbf{0 , 7 5}$ & 0,41 & 0,47 \\
\hline & $\mathrm{DO} 2$ & Aumentar a pontualidade na entrega & 5,34 & 0,57 & 0,11 & 0,47 & $\mathbf{0 , 8 0}$ & 0,46 & 0,41 \\
\hline & DO3 & $\begin{array}{l}\text { Ter CRM (Gestão do Relacionamento } \\
\text { com Clientes) confiável }\end{array}$ & 5,30 & 0,60 & 0,11 & 0,47 & 0,77 & 0,38 & 0,54 \\
\hline & DO4 & $\begin{array}{l}\text { Ter atenção às necessidades e } \\
\text { interesses dos clientes }\end{array}$ & 5,35 & 0,54 & 0,10 & 0,48 & 0,76 & 0,45 & 0,52 \\
\hline
\end{tabular}




\section{(CONTINUAÇÃO)}

\begin{tabular}{|c|c|c|c|c|c|c|c|c|c|}
\hline \multirow{4}{*}{$\begin{array}{l}\tilde{D} \\
\tilde{D}\end{array}$} & $\mathrm{GC} 1$ & $\begin{array}{l}\text { Compartilhar informação entre } \\
\text { fornecedores e a empresa para solução } \\
\text { de problemas }\end{array}$ & 5,25 & 0,66 & 0,12 & 0,42 & 0,46 & 0,75 & 0,35 \\
\hline & GC2 & $\begin{array}{l}\text { Entender que o relacionamento entre } \\
\text { fornecedor e o consumidor final é } \\
\text { crucial }\end{array}$ & 5,40 & 0,52 & 0,10 & 0,35 & 0,44 & 0,76 & 0,33 \\
\hline & GC3 & $\begin{array}{l}\text { Cumprir o cronograma de execução } \\
\text { das atividades acordado entre } \\
\text { fornecedor e consumidor final }\end{array}$ & 5,38 & 0,52 & 0,10 & 0,36 & 0,38 & 0,79 & 0,25 \\
\hline & GC4 & $\begin{array}{l}\text { Cumprir o cronograma de execução } \\
\text { financeira (orçamento) entre } \\
\text { fornecedor e consumidor final }\end{array}$ & 5,27 & 0,66 & 0,13 & 0,52 & 0,45 & $\mathbf{0 , 8 5}$ & 0,36 \\
\hline \multirow{4}{*}{$U$} & SC1 & $\begin{array}{l}\text { Ter boa impressão da prestação de } \\
\text { serviço }\end{array}$ & 5,41 & 0,53 & 0,10 & 0,39 & 0,58 & 0,33 & $\mathbf{0 , 7 8}$ \\
\hline & $\mathrm{SC} 2$ & $\begin{array}{l}\text { Estar satisfeito com o atendimento em } \\
\text { relação às reclamações }\end{array}$ & 5,39 & 0,54 & 0,10 & 0,36 & 0,45 & 0,38 & 0,74 \\
\hline & SC3 & $\begin{array}{l}\text { Avisar antecipadamente atrasos de } \\
\text { entrega de pedido }\end{array}$ & 5,35 & 0,54 & 0,10 & 0,38 & 0,44 & 0,22 & 0,75 \\
\hline & $\mathrm{SC} 4$ & $\begin{array}{l}\text { Avisar sobre garantias de serviço em } \\
\text { caso de falhas }\end{array}$ & 5,45 & 0,65 & 0,12 & 0,36 & 0,43 & 0,33 & $\mathbf{0 , 7 9}$ \\
\hline
\end{tabular}

Nota 1: KMO (Medida Kaiser-Meyer-Olkin de adequação de amostragem) $=0,88$; Teste de esfericidade de Bartlett - Aprox. Qui-quadrado - 987,559; df = 153, Significância: 0,000; Nota 2: Todas as medidas foram mensuradas em uma escala de discordância / concordância que variava de Discordo Totalmente (DT = 1) a Concordo Totalmente $(\mathrm{CT}=6)$; Nota 3: Todas as medidas foram estatisticamente significantes para $\alpha \leq 0,01$.

Fonte: dados da pesquisa

É observado pela Tabela 2 que as medidas do modelo de mensuração ficaram no lado concordante da escala com coeficiente de variação entre 0,10 e 0,17, denotando que a distribuição dos dados apresenta baixa dispersão (PRATES, 2017). Finalizada a análise da validação das medidas e escalas do modelo de mensuração, prosseguiu-se na avaliação da significância estatística das relações dos constructos do modelo.

\subsection{Avaliação do modelo estrutural do modelo de mensuração}

Os resultados são apresentados na Figura 1. 
Figura 1 - Modelo Teórico-Empírico

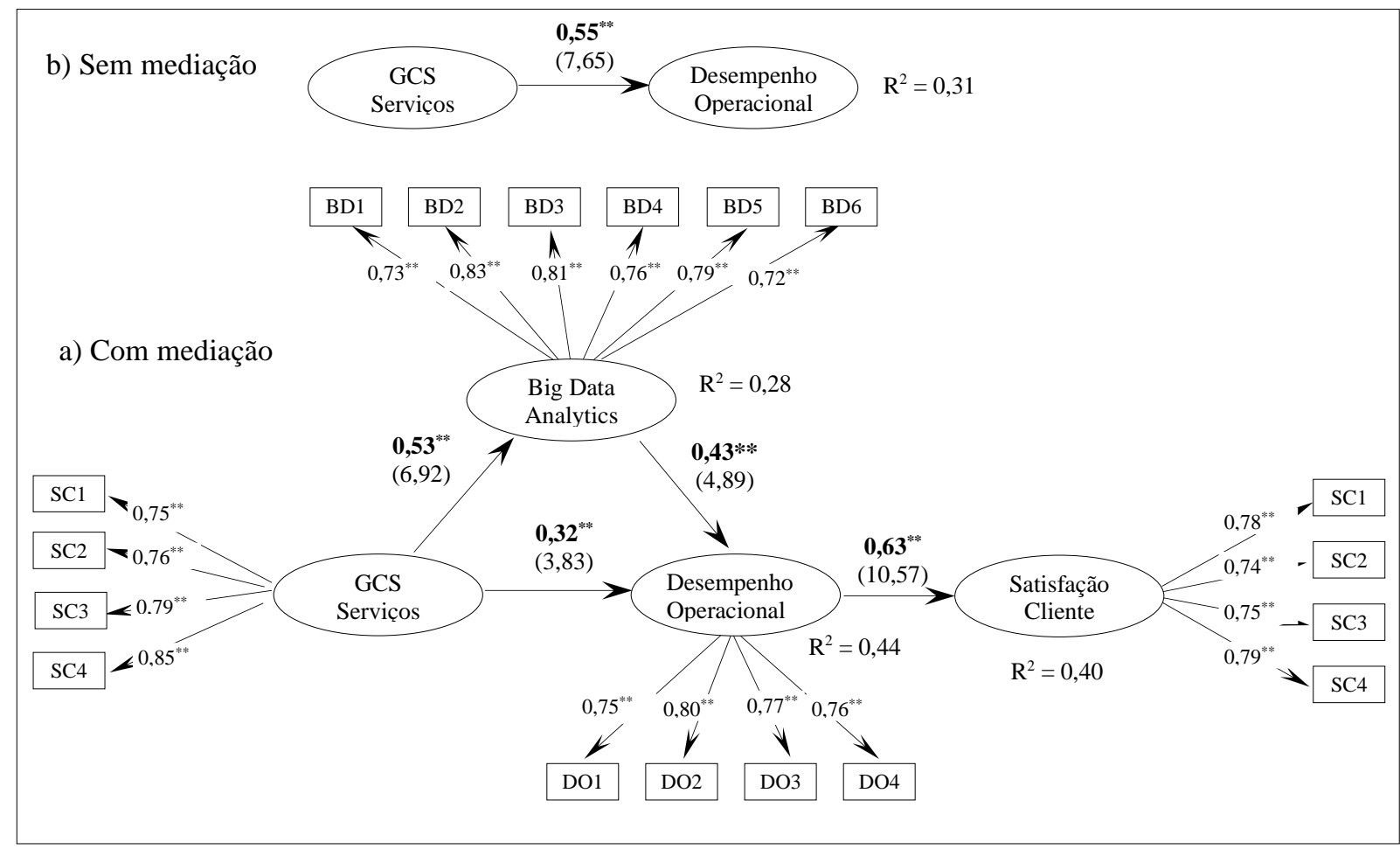

Nota: Modelo estimado pelo software SmartPLS versão 3.0 M3 e significâncias estimadas por bootstrap com n = 125 e 5000 repetições.

Fonte: dados da pesquisa

Da Figura 1 e dos dados relatados pelo software SmartPLS versão 3.0, compilou-se a Tabela 3 em que são mostrados resultados dos testes de hipóteses e significâncias estatísticas do modelo de mensuração.

Tabela 3 - Coeficientes estruturais e testes de hipótese

\begin{tabular}{lccccc}
\hline \multicolumn{1}{c}{ RELACIONAMENTO } & COEFICIENTES & ERRO & VALOR & HIPÓ- & \\
ESTRUTURAL & ESTRUTURAIS & PADRÃO & $\mathrm{t}$ & TESE & DECISÃO \\
\hline GCSS $\rightarrow$ BDA & 0,53 & 0,08 & 6,93 & $\mathrm{H}_{2}{ }^{* *}$ & Suporta \\
GCSS $\rightarrow$ Desempenho Operacional & 0,32 & 0,10 & 3,41 & $\mathrm{H}_{1}{ }^{* *}$ & Suporta \\
BDA $\rightarrow$ Desempenho Operacional & 0,43 & 0,09 & 4,82 & $\mathrm{H}_{3}^{* *}$ & Suporta \\
Desempenho Operacional $\rightarrow$ SC & 0,63 & 0,06 & 10,67 & $\mathrm{H}^{* *}$ & Suporta
\end{tabular}

Nota: $\left({ }^{* *}\right)<0,01$ : nível de significância $(\mathrm{t}>2,58) ;\left({ }^{*}\right)<0,05$ : nível de significância $(\mathrm{t}>1,96)$

Fonte: dados da pesquisa 
Deste modo, foi identificado que: a) a gestão da cadeia de suprimentos de serviços tem efeito positivo no desempenho operacional, o que suportou a hipótese $\left(\mathrm{H}_{1}\right)$, b) a gestão da cadeia de suprimentos de serviços tem efeito positivo no big data analytics, suportando a hipótese $\left(\mathrm{H}_{2}\right)$; c) o BDA (big data analytics) tem um impacto positivo no desempenho operacional, o que suportou a hipótese $\left(\mathrm{H}_{3}\right)$ e) o desempenho operacional tem impacto positivo na satisfação do cliente, o que suportou a hipótese $\left(\mathrm{H}_{4}\right)$. Todas as hipóteses foram suportadas a um nível de significância estatística de $\alpha \leq 0,01$.

Para verificar a mediação do BDA (big data analytics) na relação entre a GCSS e o DO, foi aplicado o teste da variância contabilizada (VAF) apresentado na Equação [2], cujos valores extraídos foram: $\beta_{12}=0,53 ; \beta_{23}=0,43$ e $\beta_{13}=0,32$. Obteve-se o valor VAF $=\left[\frac{0,53 \times 0,43}{(0,53 \times 0,43)+0,32}\right]$ $=0,42$, dentro do intervalo $0,20 \leq \mathrm{VAF} \leq 0,80$, que, de acordo com a abordagem de Hair et al. (2014), aponta para um efeito da mediação parcial.

Portanto, o BDA (big data analytics) medeia, parcialmente, a relação entre gestão da cadeia de suprimentos de serviços e desempenho operacional, suportando a hipótese $\left(\mathrm{H}_{1 \mathrm{a}}\right)$.

Para avaliar o ajuste do modelo, inicialmente, utilizou-se do índice de adequação GoF (Godness of Fit), dado pela média geométrica (da raiz quadrada do produto de dois indicadores) entre o R $\mathrm{R}^{2}$ médio (adequação do modelo estrutural) e a média ponderada das VME (adequação do modelo de mensuração) (HAIR et al., 2014). Dado que a média de VME foi 0,60 [0,60 + $0,59+0,62+0,58) / 4=0,60]$ e a média de $\mathrm{R}^{2}$ foi $0,37[(0,28+0,44=0,40) / 3=0,37]$, obtevese valor GoF de $0,47[\mathrm{GOF}=\sqrt{0,60 * 0,37}=0,47]$. Esse valor levou a se considerar o modelo adequado aos dados coletados.

Como complemento, verificou-se a validade preditiva $\left(\mathrm{Q}^{2}\right)$ para avaliar quanto o modelo apresentado estava próximo àquilo que era esperado. Referiu-se à qualidade da predição do modelo, ou acurácia do modelo ajustado (HAIR et al., 2014). Valores maiores que zero são obtidos como critério de avaliação (HAIR et al., 2014). A perfeição de dado modelo ocorreria para $\mathrm{Q}^{2}=1$, indicando que o modelo reflete a realidade sem erros (HAIR et al., 2014).

Quanto ao Efeito do Tamanho da Amostra (f²), a medição ocorre por meio da inclusão e exclusão dos construtos do modelo. Procura-se avaliar o quando cada construto é útil para o ajuste do modelo (HAIR et al., 2014). Os valores de 0,02, 0,15 e 0,35 são considerados pequeno, médio e grande, respectivamente (HAIR et al., 2014). Os valores obtidos para a validade preditiva $\left(\mathrm{Q}^{2}\right)$ e o tamanho do efeito $\left(\mathrm{f}^{2}\right)$ são mostrados na Tabela 4. 
Tabela 4 - Valores dos indicadores da validade preditiva $\left(\mathrm{Q}^{2}\right)$ e do tamanho do efeito $\left(\mathrm{f}^{2}\right)$

\begin{tabular}{lcc}
\hline CONSTRUCTO & CV RED $\left(\mathrm{Q}^{2}\right)$ & CV COM $\left(\mathrm{f}^{2}\right)$ \\
\hline Gestão da Cadeia de Suprimentos de Serviços & 0,36 & 0,36 \\
Big Data Analytics & 0,15 & 0,44 \\
Desempenho Operacional & 0,24 & 0,32 \\
Satisfação do Cliente & 0,20 & 0,31 \\
\hline \multicolumn{1}{c}{ Valores de referência } & $0,02=$ pequeno efeito \\
& $\mathrm{Q}^{2}>0$ & $0,15=$ médio efeito \\
& & $0,35=$ grande efeito
\end{tabular}

Fonte: dados da pesquisa

Da Tabela 4, entende-se que tanto os valores de $\mathrm{Q}^{2}$, como os valores de $\mathrm{f}^{2}$, indicam que o modelo apresentado tem precisão e que os respectivos construtos são, de fato, importantes para o ajuste geral do modelo.

Para melhor sintetizar os resultados da análise do modelo de caminhos (path model analysis), os efeitos direto, indireto e total entre os construtos são apresentados na Tabela 5.

Tabela 5 - Efeitos direto, indireto e total entre os construtos do modelo de mensuração

\begin{tabular}{ccc|ccc|ccc}
\hline & \multicolumn{2}{c}{ BDA } & \multicolumn{3}{c|}{ DO } & \multicolumn{3}{c}{ SC } \\
\cline { 2 - 9 } CONSTRUCTOS & \multicolumn{2}{c|}{ Efeitos } & \multicolumn{3}{c|}{ Efeitos } & \multicolumn{3}{c}{ Efeitos } \\
\cline { 2 - 9 } & Direto & Total & Direto & Indireto & Total & Direto & Indireto & Total \\
GCSS & 0,53 & 0,53 & 0,32 & 0,23 & 0,55 & 0 & 0,35 & $\mathbf{0 , 3 5}$ \\
BDA & 0 & 0 & 0,43 & 0 & 0,43 & 0 & 0,27 & $\mathbf{0 , 2 7}$ \\
DO & 0 & 0 & 0 & 0 & 0 & 0 & 0,63 & $\mathbf{0 , 6 3}$ \\
\hline
\end{tabular}

Fonte: dados da pesquisa.

Conforme se observou pela Tabela 5, o desempenho operacional apresentou o maior impacto na satisfação do cliente $(0,63)$, seguido da $\operatorname{GCSS}(0,35)$ e do $\operatorname{BDA}(0,27)$. 


\section{CONCLUSÕES E SUGESTÕES PARA PROSSEGUIMENTO}

O estudo mostrou que o BDA medeia parcialmente a relação entre a GCSS e o Desempenho Operacional, por sua vez, influencia na Satisfação do Cliente. Todavia, esse resultado traz implicações para a teoria e para a prática administrativa conforme descrito a seguir.

a) Teoria. $\mathrm{Na}$ perspectiva da vantagem competitiva (PORTER, 1985), o BDA reduz os estoques de segurança, contribuindo para maior acuracidade na previsão de demanda e no monitoramento de desempenho dos fornecedores. Os processos na cadeia de suprimentos de serviços se tornam mais automatizados e, como resultado, tarefas tradicionais são parcialmente substituídas, com agregação de valor. Como consequência, a transição do papel tradicional da gestão da cadeia de suprimentos de serviços aumentará a importância da intuição humana e confiança para tomada de decisões estratégicas.

Na perspectiva da teoria dos custos de transação (WILLIAMSON, 1999), o BDA (big data analytics) ajudará a reduzir a assimetria da informação, evitando o comportamento póscontratual em que uma parte possui uma informação privada e pode dela tirar proveito em prejuízo de sua contraparte. Nesse caso, o uso do BDA possibilitará a utilização da informação de modo imediato, sem que seja necessário um fluxo de informações até uma instância decisória central.

$\mathrm{Na}$ perspectiva da visão relacional, as rendas proporcionadas pelas relações de trocas poderão ser melhoradas, se comparadas com o que poderia ser gerado isoladamente (DYER; SINGH, 1998). A adoção de aplicações de BDA (big data analytics) está se tornando relevante para o domínio da gestão da cadeia de suprimentos de serviços. Os bancos de dados conterão muito valor e as empresas poderão coletar uma variedade de informações, em profundidade, e analisá-las apropriadamente por meio do BDA (big data analysis). Nesse sentido, a vantagem competitiva poderá provir do melhor gerenciamento das relações com os fornecedores e não exclusivamente do foco nos clientes.

Portanto, nas perspectivas dessas três linhas de pensamento teórico, de forma geral, as literaturas ainda são incipientes, de tal modo que existe um amplo campo para aprofundamentos acadêmicos e científicos. 
Acredita-se que novas pesquisas neste campo de estudo sejam capazes de trazer significativas contribuições, tanto para as práticas gerenciais, quanto para as práticas acadêmicas.

b) Práticas administrativas. É consenso que prestar serviços no mercado condominial é uma atividade tão complexa como em outras diversas áreas do mercado de serviços. A atenção ao relacionamento da cadeia de suprimentos permite que a organização se posicione de modo estratégico em relação aos seus concorrentes e seja lembrada positivamente pelos seus clientes e parceiros na prestação de serviços de excelência. Fatores como o compartilhamento e troca de informações, recursos, o cumprimento de cronogramas financeiros e de execução de serviços contribuirão para um relacionamento com ganhos mútuos entre fornecedor e cliente.

De modo geral o estudo permitiu verificar que o uso do BDA é eficaz como uma tecnologia digital que permite uma melhor análise das informações por parte do fornecedor de serviços quanto a fatores como personalização, customização dos clientes, processos organizacionais, monitoramento de processos e até mesmo equipamentos que fazem parte da rotina dos condomínios e que são indispensáveis para o funcionamento e bem-estar dos condôminos. As empresas que têm utilizado o BDA, de modo a otimizar os seus processos, têm conseguido significativas melhorias em seu desempenho operacional e, como consequência, satisfazer as necessidades do cliente.

Os sistemas de comunicação, monitoramento, controle e automatização de dispositivos são capazes de conectar-se com dispositivos de modo a contribuir para soluções empresariais e condominiais e comercialização de consumo de água, gás, energia elétrica de sistemas de iluminação, geradores de energia, elevadores, ar condicionado, controle de acesso, circuito fechado de TV, controle de alarme de incêndio, quadros elétricos, qualidade do ar, temperatura, pressão, entre outros pontos (CONECTON, 2017).

Sendo assim, não se deve descartar a tecnologia BDA, pois trata-se de uma ferramenta fundamental para facilitar a vida e os processos das empresas inseridas em uma sociedade moderna. Uma vez que o mercado condominial ainda é carente de inovações tecnológicas, se faz necessário um aprofundamento do tema de modo que os síndicos, gestores condominiais, conselheiros, moradores e prestadores de serviços estejam atentos a essa ferramenta disponível no mercado. 
A utilização do $B D A$ permite que as empresas de prestação de serviços condominiais busquem o desenvolvimento de ações tecnológicas e apliquem tais inovações em suas rotinas operacionais para tornarem-se diferenciadas no mercado e, consequentemente, proporcionarem satisfação aos clientes. Acredita-se que o BDA é uma importante ferramenta para a gestão da cadeia de suprimentos de serviços e para o desempenho operacional das organizações. À medida que os prestadores de serviços condominiais entendam esta relação, e se disponham a utilizar a ferramenta como facilitadora de seus processos de prestação de serviços, poderão melhor se desenvolver e satisfazer os seus clientes.

Portanto, com base no resultado obtido, implicações teóricas, implicações para a prática administrativa e contribuição, pode-se concluir que o BDA é uma importante ferramenta de gestão por facilitar a execução das atividades de prestação de serviços em condomínios residenciais no sentido de se antever possíveis problemas em equipamentos utilizados pelos clientes em elevadores, bombas hidráulicas, portões automáticos e câmeras de monitoramento de acesso de pessoas nos condomínios, melhorando assim, o desempenho operacional das empresas prestadoras de serviços condominiais, e se estendendo para a satisfação do cliente pela prestação do serviço.

Por fim, sugere-se para prosseguimento do estudo o desenvolvimento de novas pesquisas, por exemplo, utilizando o BDA como fator moderador, de tal modo a melhor identificar relações entre a GCSS e o DO. Investigar outros construtos relacionados a este tema que venham prestar um melhor entendimento do assunto certamente traria excelentes contribuições para as práticas acadêmicas e gerenciais.

\section{REFERÊNCIAS}

ANDERSON, E. W. Customer satisfaction and word-of-mouth. Journal of Service Research, v. 1, n. 1, p. 5-17, 1998. <https://doi.org/10.1177/109467059800100102>

BALLOU, R. H. The evolution and future of logistics and supply chain management. European Business Review, v. 19, n. 4, 2007.

BALTACIOGLU, T.; ADA, E.; KAPLAN, D. M.; YURT, O.; KAPLAN, C. A new framework for service supply chains. The Service Industries Journal, v. 27, n. 2, 2007.

BLOEMER, J. M. M.; KASPER, H. D. P. The complex relationship between consumer satisfaction and brand loyalty. Journal of Economic Psychology, v. 16, n. 2, p. 311-329, 1995. 
BOWERSOX, D. J.; CLOSS, D. J.; COOPER, M. B. Gestão da cadeia de suprimentos e logística. 2. Ed. Rio de Janeiro: Elsevier, 2007.

BROWN, S.; LAMMING, R.; BESSANT, J.; JONES, P. Administração da produção e operações. Rio de Janeiro: Editora Campus / Elsevier, 2006.

CAO, M.; ZHANG, Q. Supply chain collaboration: Impact on collaborative advantage and firm performance. Journal of Operations Management, v. 29, n. 3, 2011, p. 163-180. <https://doi.org/10.1016/j.jom.2010.12.008>

CARVALHO, M. F. A Importância da Informação no Desempenho da Cadeia de Suprimentos - Um Estudo Exploratório. In: XII SIMPEP. Anais... Bauru, SP, Brasil, 07 a 9 de novembro de 2005 .

CHEN, C. P.; ZHANG, C. Y. Data-intensive applications, challenges, techniques and technologies: A survey on Big Data. Information Sciences, 275, 314-347, 2014.

CHIN, W. W. Issues and Opinion on Structural Equation Modeling. MIS Quarterly Management Information Systems Research Center. University of Minnesota, v. 22, n. 1, p. vii-xvi, Mar. 1998.

CHOI, T. M.; LAMBERT, J. H. Advances in risk analysis with big data. Risk Anal, 37, p. 1435-1422, 2017.

CHOU, M. C. et al. Analysis of a software-focused products and service supply chain. IEEE Transactions on Industrial Informatics, v. 2, n. 4, 2006.

COHEN, J. Statistical Power Analysis for the Behavioral Sciences. 2. Ed. New York: Psychology Press, 1988.

COMBS, J. G.; CROOK, T. R.; SHOOK, C. L. The dimensionality of organizational performance and its implications for strategic management research. Research Methodology in Strategy and Management, v. 2, n. 5, p. 259-286, 2005.

CONECTON. Consumo de água, gás, energia, controle de iluminação e muito mais. 2017. Disponível em <http://conectcon.com/2017/04/17/automacao-condominios/>. Acesso em: 20/05/2018.

COSTA, M. R.; TORRES, N. Gestão da cadeia de suprimentos de serviços: uma análise das atividades operacionais logísticas de empresas exibidoras de filmes de longa-metragem de Belo Horizonte. GEPROS. Gestão da Produção, Operações e Sistemas, v. 9. n. 3, p. 61-78, 2014.

DILLMAN, D. A. Mail and internet surveys: the tailored design method. John Wiley \& Sons, Inc. 2th edition. 464p. 2000.

DONALD J. B.; DAVID J. C.; COOPER M. B.; JOHN C. B. Gestão logística da cadeia de suprimentos. Porto Alegre: AMGH, 4. Ed., 2014.

DYER, J. H.; SINGH, H. The relations view: co-operative strategy and sources of inter- 
organizational competitive advantage. Academy of Management Review, v. 23, p. 660-679, 1998.

ELLRAM, L. M.; TATE, W. L.; BILLINGTON, C. Understanding and managing the services supply chain. Journal of Supply Chain Management, v. 40, n. 4, 2004.

FAVARETTO, F. Gerenciamento de informações em cadeias de prestações de serviços. Perspectivas em gestão \& conhecimento, v. 2, n. 1, p. 3-20, 2012.

FLYNN, B. B.; HUO, B.; ZHAO, Z. The impact of supply chain integration on performance: A contingency and configuration approach. Journal of Operations Management, v. 28, p. 58$71,2010$.

HAIR, J. F.; HUlT, G. T.; RINGLE, C. M.; SARSTEDT, M. A Primer on Partial Least Squares Structural Equation Modeling (PLS-SEM). Los Angeles: Sage Publications, 2014.

ITTMANN, H. W. The Impact of big data and business analytics on supply chain management. Journal of Transport and Supply Chain Management, v. 9, n. 1, p. 1-9, 2015.

KIRON, D.; PRENTICE, P. K.; FERGUSON, R. B. The analytics mandate. MIT Sloan Management Review, v. 55, n. 4, p. 1-25, 2014.

KOTLER, P. Administração de Marketing. 10 ed. São Paulo: Pearson Education do Brasil, 2000 .

KRITHIKA, V; KAUR, A.; SEKARAN, C.; RAJENDRAN, C. Developing a conceptual relationship between web service supply chain entities. In: IEEE World Congress on Services. Proceedings... 2011.

LI, B.; LI, Y. Internet of things drives supply chain innovation: A research framework. The International Journal of Organizational Innovation, v. 9, n. January 2017, p. 71-93, 2017.

MAJEED, M. A. A.; RUPASINGHE, T. D. Internet of things (IoT) embedded future supply chains for industry 4.0: An assessment from an ERP-based fashion apparel and footwear industry. International Journal of Supply Chain Management, v. 6, n. 1, p. 25-40, 2017.

MALHOTRA, N. K. Pesquisa de Marketing: Uma Orientação Aplicada. Porto Alegre: Bookman, $7^{\text {a }}$ edição. 800p. 2019

MANYIKA, J.; CHUI, M.; BROEN, B.; BUGHIN, J.; DOBBS, R.; ROXBURGH, C.; BYERS, A. H. Big Data: the next frontier for innovation, competition, and productivity, 2011.

MILAN, G. S.; DE TONI, D. A. A construção de modelo sobre a retenção de clientes e seus antecedentes em um ambiente de serviços. Revista Eletrônica de Administração, v. 72, n. 2, p. 433-467, 2012.

MILAN, G. S.; EBERLE, L.; CORSO, A.; TONI, D. A Qualidade em Serviços e a Satisfação de Clientes: Comparação entre a percepção de alunos de graduação e de pós-graduação de uma IES. Revista de Administração UFSM, v. 8, n. 3, p. 415-437, 2015. 
MILLER, J. G; ROTH, A. V. A taxonomy of manufacturing strategies. Management Science, v. 40, n. 3, p. 285-304, 1994.

MÜLLER, S. D.; JENSEN, P. Big data in the Danish industry: application and value creation. Business Process Management, v. 23, n. 3, p. 645-670, 2017.

PAULRAJ, A.; CHEN, I. J. Strategic buyer-supplier relationships, information technology and external logistics integration. The Journal of Supply Chain Management, p. 3, 2007.

PIRES, S. R. I. Gestão da cadeia de Suprimentos: conceitos, estratégias, práticas, e casos Supply Chain Management. São Paulo: Editora Atlas, 2a . Edição, 7. Reimpressão, 2013.

PIRES, S. R. I.; CARRETERO-DÍAZ, L. E. Gestión de la cadena de suministros. McGraw Hill, Madrid, 2007.

PORTER, M. E. Competitive Advantage. New York. The Free Pass, 1985.

PORTER, M. E.; HEPPELMANN, J. E. How Smart, Connected Products are Transforming Companies. Harvard Business Review. October 2015.

PRATES, W. O. Estatística para ciências sociais aplicadas I. Salvador: UFBA, 156p., 2017.

RODRIGUEZ, L.; CUNHA, C. Impacts of Big Data Analytics and Absorptive Capacity on Sustainable Supply Chain Innovation. A Conceptual Framework. LogForum, v. 14, n. 2, p. 151-161, 2018.

ROSENZWEIG, E. D.; EASTON, G. S. Tradeoffs in manufacturing? A Meta-analysis and critique of the literature. Production and Operations Management, v. 19, p. 127-141, 2010.

ROSSMANN, B.; CANZANIELLO, A.; GRACHT, H.; HARTMANN, E. The future and social impact of big data analytics in supply chain management: results from a Delphi study. Technological Forecasting \& Social Change, v. 130, p. 135-149, 2018.

SANDERS, N. R. How to use big data to drive your supply chain. California Management Review, v. 58, n. 3, 2016.

STARR, M. K. Changing agendas for operations management. Gestão \& Produção, v. 12, n. 13, São Carlos, 2005.

SUKATI, I.; HAMID, A. B.; BAHARUN, R.; YUSOFF, R. M. The study of supply chain management strategy and practices on supply chain performance. Procedia - Social and Behavioral Sciences, v. 40, p. 225-233, 2012.

TONI, D.; MAZZON, J. A. Teste de um modelo teórico sobre o valor percebido do preço de um produto. Revista de Administração, v. 49, n. 3, p. 549-565, 2014.

WAMBA, S. F.; GUNASEKARAN, A.; AKTER, S.; REN, S.; DUBEY, R.; CHILDE, S. Big data analytics and firm performance: Effects of dynamic capabilities. Journal of Business Research, v. 70, 2017, p. 356-365. <http://dx.doi.org/10.1016/j.jbusres.2016.08.009> 
WANG, Y.; WALLACE, S. W.; SHEN, B.; CHOI, T. Service supply chain management: A review of operational models. European Journal of Operational Research, v. 247, p. 685698, 2015.

WETZELS, M.; ODEKERKEN-SCHRÖDER, G.; OPPEN, C. VAN. Using PLS Path Modeling for Assessing Hierarchical Construct Models: Guidelines and Empirical Illustration. MIS Quarterly, v. 33, n. 1, p. 177-195, 2009.

WILLIAMSON, O. E. Strategy Research: governance and competence perspective. Strategic Management Journal, v. 20, n. 12, p. 1087-10108, 1999.

ZEITHAML, V. A.; BITNER, M. Marketing de Serviços: a empresa com foco no cliente. Porto Alegre: Bookman, $2^{\mathrm{a}}$ edição, 2003. 